\title{
Las actitudes del alumnado hacia el aprendizaje del inglés como idioma extranjero: estudio de una muestra en el sur de España
}

\author{
DIEGO URIBE \\ Los Angeles Southwest College \\ José GuTIÉRRez y DANIEl MADrid \\ Universidad de Granada
}

Recibido:12 agosto 2007 / Aceptado: 9 enero 2008

ISSN: $1697-7467$

\begin{abstract}
RESUMEN: Este estudio investiga las actitudes hacia el aprendizaje del inglés como idioma extranjero y si esas actitudes se ven afectadas por el sex, nota media en inglés, clases particulares, clase social y curso de iniciación al inglés. Los participantes fueron 177 alumnos matriculados en la Enseñanza Secundaria Obligatoria en cuatro institutos del sur de España. Desarrollamos el cuestionario a partir de los puestos en práctica por Gardner (1985), Jakobobitz (1970) y Madrid (1999). Los resultados muestran que las actitudes de esta muestra hacia el inglés son ligeramente positivas. No se encontraron correlaciones importantes entre actitudes y variables independientes. Sugerimos una explicación: el alumnado en las comunidades autónomas de Andalucía y Murcia aprende el inglés en contextos académicos sin interaccionar regularmente con la comunidad del otro idioma.
\end{abstract}

Palabras clave: actitudes, diferencias individuales, aprendizaje de idiomas extranjeros, contexto cultural.

\begin{abstract}
This study examines the attitudes towards learning English as a foreign language and whether those attitudes are moderated by gender, average grade in English, private tutoring, social class, and grade they started to study English. Participants were 177 Compulsory Secondary Education students in four schools in the South of Spain. The measures used to assess attitudes were modeled after previous questionnaires by Gardner (1985), Jakobobitz (1970), and Madrid (1999). Results show that the attitudes of this sample towards English are slightly positive. No important correlations were found between attitudes and independent variables. One possible explanation is suggested: students in the autonomous communities of Andalusia and Murcia learn English in academic settings without regularly interacting with the target language community.
\end{abstract}

Key words: attitudes, individual differences, foreign language learning, cultural context.

\section{INTRODUCCIÓN}

La necesidad de dominar un idioma extranjero en las sociedades contemporáneas es un hecho ineludible, una cuestión de urgencia y hasta de supervivencia. La ruptura de las fronteras físicas y culturales ha cambiado las reglas del juego y ha difuminado las barreras de 
comunicación entre los pueblos. El mundo se ha convertido en una aldea global donde los ciudadanos de cualquier país sienten la necesidad de comunicarse con personas de otras culturas y nacionalidades, ya sea por razones artísticas, económicas, políticas o científicas. Ser ciudadano hoy supone disponer de recursos de comunicación orales y escritos diferentes a los de nuestra cultura de origen. De lo contrario, nuestras posibilidades para encontrar trabajo, disfrutar de nuestro tiempo de ocio o debatir sobre problemas contemporáneos no serán las mismas. El alumnado ha percibido desde hace tiempo la importancia de aprender un segundo idioma en la sociedad actual, ya que, piensa, le será de mucha utilidad en el futuro (Antes, 1999). En España, de acuerdo con Comisión Europea (2006), apenas el 3 por ciento domina el inglés correctamente, aunque el 36 por ciento declara, según otro estudio de la Commission of the European Languages (2005), que puede participar en una conversación en inglés, muy por debajo de la media europea que es del 50 por ciento.

La importancia de aprender un idioma se manifiesta de manera diferente en cada persona y al profesorado se nos plantean muchas dudas ante el hecho educativo, lo que hace que tengamos diferentes expectativas para el grupo en la clase (Eccles y Wigfield, 1985; Good y Brophy, 2003; Jussim, Smith, Madon y Palumbo, 1998; Pellegrini y Blachtford, 2000; RubieDavies, 2006). A veces, no entendemos bien su comportamiento y la distancia que normalmente existe entre ambos suele evitar un mejor conocimiento de sus formas de proceder y la naturaleza de éstas, lo que se convierte en un obstáculo para el buen funcionamiento de la clase de inglés. En muchas ocasiones, tenemos la impresión de que existe cierta desgana por su parte ante los diferentes mensajes que les enviamos y que sus actuaciones ante las diversas actividades y ejercicios no se desarrollan de la manera que quisiéramos.

Cuando el alumnado viene a nuestras aulas por primera vez, lo hace con numerosos prejuicios acerca del inglés (es lo que Hosenfeld, [1978], denominó miniteorías del aprendizaje de un idioma.) debido a varias razones: ya sea por experiencias pasadas, por el entorno que les rodea o por cualquier otro tipo de cuestión que puede, incluso, distorsionar su progreso en el idioma (Mantle-Bromley, 1995). Esta investigación se va a centrar en las diferencias actitudinales al aprender un idioma extranjero. Fue allá en los años 70 cuando se pusieron los cimientos de lo que con gran acierto se ha desarrollado posteriormente, una época más conocida como la década del alumnado, donde los factores cognitivos, afectivos y sociológicos adquirieron la importancia que siempre debieron tener como elementos primordiales en el aprendizaje de idiomas.

Es esta diversidad de factores personales que entran en juego en el proceso educativo la que hace que algunos tengan más éxito que otros a la hora de aprender un idioma, no importa que la experiencia haya sido la misma, y que ciertos aspectos sean más fáciles de asimilar que otros (Bialystok, 1978). Estos factores personales o diferencias individuales, se refieren a las «dimensiones de las características personales que afectan al alumnado en mayor o menor medida» (Dörnyei, 2006:42). Según el mismo autor, las cinco diferencias individuales más importantes dentro del campo del aprendizaje de segundos idiomas son: personalidad, aptitud, motivación -y, por tanto, las actitudes-, estilos y estrategias de aprendizaje. A partir de aquí, se nos plantean una serie de interrogantes: ¿Cuál es la naturaleza de las diferencias en el rendimiento del alumnado cuando se enfrentan al estudio de un idioma extranjero? ¿Son las personas tímidas peores aprendiendo idiomas? ¿Es lo mismo aprender un idioma para una persona que pertenezca a un grupo étnico minoritario que para un individuo de un grupo mayoritario? La importancia de estas preguntas es cada día mayor, no sólo para la investi- 
gación, sino también para el profesorado de idiomas, y las implicaciones sociales, psicológicas y educativas en el aprendizaje de un idioma es algo que viene de lejos; pues es cierto que en algunos casos el aprendizaje de un idioma es una experiencia relativamente corta a la que se enfrenta el alumnado durante su educación, mientras que en otros es un camino hacia el bilingüismo.

Hoy en día casi todos los investigadores están de acuerdo con la importancia de los factores afectivos en el aprendizaje de un idioma (Gardner, 1985; Masgoret y Gardner, 2003; Gardner, Masgoret, Tennant y Mihic, 2004); es decir, aquellas características emocionales de las personas que influyen en la manera en que éstas responden a una determinada situación (Gardner y MacIntyre, 1993). Aunque anteriormente algunos como Arsenian (1945) habían incidido en la relevancia de las actitudes, sería Lambert (1955) quien verdaderamente puso las bases para el desarrollo de este campo. En su primer trabajo con un grupo de estudiantes americanos, Lambert postulaba la existencia de dos tipos diferentes de orientaciones: instrumentales e integrativas. Las primeras denotan un uso pragmático de la lengua y su objetivo final no es la integración, sino el reconocimiento social y las ventajas económicas. Mientras que las segundas conllevan actitudes positivas hacia la comunidad del otro idioma, deseo de formar parte de ella y relacionarse con sus hablantes (Lasagabaster, 2002). A partir de ese momento, empezaron a proliferar un gran número de estudios que Masgoret y Gardner (1999) resumen afirmando que «las actitudes juegan un papel fundamental en el aprendizaje de idiomas, ya que fortalecen la motivación de las personas para aprenderlos» (pág. 220). Los dos tipos de actitudes que más se han investigado han sido las actitudes hacia el aprendizaje de un idioma y actitudes hacia la comunidad de ese idioma. Mientras las primeras parecen claramente educativas, las segundas denotan un aspecto puramente social.

En el ámbito de lenguas extranjeras, Manzaneda y Madrid (1997) puntualizan que el concepto de actitudes es muy complejo, engloba componentes cognitivos, afectivos, conativos y evaluativos, y creen que se manifiesta mediante: a) un conjunto de creencias y opiniones sobre la enseñanza y aprendizaje de la lengua extranjera; b) determinadas reacciones y tonos emocionales a lo largo de esos procesos de enseñanza/aprendizaje de la lengua extranjera; c) cierta predisposición o tendencia a comportarse de varias formas en las diferentes situaciones de clase; y d) una valoración positiva, negativa o indiferente respecto a esas situaciones de enseñanza/aprendizaje de la lengua extranjera.

En Europa, el inglés es el principal vehículo de comunicación para los europeos, aunque todavía existen diferencias de uso entre norte y sur (Cenoz, 2003). Según esta autora, se debe estudiar el contexto educativo en el que tiene lugar el aprendizaje, ya que hoy día en España, por citar un ejemplo, más de un 34 por ciento tiene dos lenguas oficiales (Lasagabaster, 2002). Lo que si parece claro, según Cenoz, es la importancia de la introducción al inglés a temprana edad, pues no importa si se trata del segundo o del tercer idioma, no hay efectos negativos en el desarrollo cognitivo y lingüístico del alumnado, y sus actitudes tienden a ser, por lo general, más positivas que las de los mayores. Otros estudios en el contexto español y europeo llegaron a las mismas conclusiones que Cenoz sobre la importancia de empezar temprano a aprender inglés (Agullo, 2006; Artigal, Arizmendi y Azpillaga, 1996; Gálvez Díaz, 2001; Littlewood, 2001; Mur, 1998; Rodríguez Sánchez y McCarthur, 2001).

No se han encontrado grandes diferencias (Gardner, 2007) en los ciudadanos europeos entre motivaciones integrativas o motivaciones instrumentales a la hora de aprender un segundo idioma. Además, no importa que las actitudes hacia el inglés sean positivas ni que su 
introducción sea temprana, diferentes investigaciones indican que la mayoría de los europeos necesita mucho aliento para aprender inglés (Comisión Europea, 2006) y llega a la universidad con un nivel oral poco aceptable (Ruíz Garrido, 2007). Algunos achacan el problema a las diferencias metodológicas que existen de unos lugares a otros y al escaso tiempo que se dedica a su aprendizaje (Rodríguez López y Varela Méndez, 2004).

\section{MÉTODo}

\subsection{Objetivo del trabajo y variables}

La intención de este estudio es conocer las actitudes del alumnado en el sur de España (en las comunidades autónomas de Andalucía y Murcia) hacia el profesorado de inglés, el libro de texto de inglés, la clase de inglés y las actividades en inglés; establecer la relación entre su actitudes y el entorno inmediato; y estudiar la influencia que tienen variables como las clases particulares, curso de iniciación al inglés, nota media en inglés, sexo y clase social en el proceso actitudinal.

\subsection{Participantes}

El grupo que participó en este estudio cursaba $3^{\circ}$ y $4^{\circ}$ de la ESO en cuatro institutos públicos de educación secundaria de las provincias de Granada, Almería y Murcia, y con edades comprendidas entre los 14 y 17 años. 177 participantes (90 chicas y 87 chicos) respondieron al cuestionario sobre actitudes, mientras que la muestra para los análisis de la varianza fue inferior (género, $\mathrm{n}=176$; curso de iniciación, $\mathrm{n}=151$; clase social, $\mathrm{n}=133$; nota media, $n=167$; clases particulares, $n=133$ ). La diferencia se explica en base a que las cuatro subescalas eran opcionales. Creemos que la muestra que participó en nuestro trabajo representa un grupo estándar en el ámbito de la Enseñanza Secundaria Obligatoria en las comunidades autónomas de Andalucía y Murcia. Estos cuatro institutos públicos utilizaban el mismo libro y parecidos materiales curriculares complementarios, tenían una población inmigrante poco significativa y estaban localizados en núcleos urbanos de entre 3 y 30 mil habitantes; además de tener similares directrices educativas por parte de sus respectivas comunidades autónomas. El tipo de muestreo utilizado fue un muestreo por conveniencia.

\subsection{Procedimiento}

Cuatro subescalas sirvieron para medir las actitudes del alumnado hacia el aprendizaje del inglés como idioma extranjero basadas principalmente en modelos puestos en práctica por Gardner (1985), Jakobobitz (1970) y Madrid (1999). A estas cuatro subescalas, que nosotros hemos denominado ACTIPROF (Actitudes hacia el profesor/la profesora de inglés), ACTICLAS (Actitudes hacia la clase de inglés), ACTIWORK (Actitudes hacia los ejercicios en inglés) y ACTITEXT (Actitudes hacia el libro de texto de inglés), se le incluyó una sección en la que había que contestar a una serie de preguntas. Antes de suministrarlos a los grupos elegidos, se llevó a cabo un estudio piloto. Esto sirvió para hacer ciertas correcciones como eliminar el vocabulario que parecía más difícil, modificar varios enunciados y suprimir una quinta 
escala. Asimismo, antes de la aplicación del cuestionario se explicó al alumnado el significado de seis palabras, procedimiento este que realizamos de idéntica manera con todos los grupos. El tiempo que tardó el alumnado en responder a todos los ítems (de $0=$ Muy negativa a 4 = Muy positiva) de las subescalas fue alrededor de una hora.

\subsection{Análisis de los datos}

En primer lugar se hallaron los valores de fiabilidad de la muestra, a partir del cálculo de la prueba a de Cronbach:

Tabla 1. Valores de fiabilidad de la muestra según la prueba a de Cronbach.

\begin{tabular}{lc}
\hline Cuestionario & $\alpha$ de Cronbach \\
\hline Subescala ACTIPROF (17 ítems) & .86 \\
Subescala ACTICLAS (19 ítems) & .85 \\
Subescala ACTITEXT (16 ítems) & .87 \\
Subescala ACTIWORK (21 ítems) & .88 \\
Cuestionario Global (73 ítems) & .96 \\
\hline
\end{tabular}

Los valores de fiabilidad obtenidos globalmente para esta muestra, y un conjunto de 73 ítems, son considerablemente altos $(\alpha=.96)$; significa que se puede confiar plenamente en los resultados obtenidos con este conjunto de instrumentos de medida, dado que el número de ítems es elevado y mantiene una fuerte consistencia interna en los aspectos medidos parcialmente en las cuatro subescalas.

\section{Resultados}

\subsection{Cuestionario}

Se realizó un estudio de la información recogida en las cuatro subescalas de estimación. Los resultados que se observan en la Figura 1 corresponden a los valores medios obtenidos en la muestra en cada una de ellas.

Como se observa, la actitud del alumnado oscila entre 3 y 4 , con una media global de 3.45 (resultado del total de la suma de los valores obtenidos en las cuatro subescalas empleadas). Este valor representa, globalmente, una actitud entre indiferente y positiva; es decir, ni muy alta ni muy baja. Un análisis específico de las medias parciales de cada una ratifica nuevamente la anterior observación con valores comprendidos en el intervalo 3 y 4: ACTICLAS $($ Media $=3.50)$, ACTIPROF $($ Media $=3.58)$, ACTITEXT $($ Media $=3.38)$ Y ACTIWORK $($ Media $=3.37)$.

Todo lo anterior pone de manifiesto que el perfil medio-tipo de las percepciones que muestra el alumnado español respecto al conjunto de factores condicionantes del aprendizaje del inglés no es ni excesivamente positivo ni excesivamente negativo, con una cierta tendencia más hacia lo positivo que a lo indiferente. 
Figura 1. Valores medios de las cuatro subescalas empleadas.

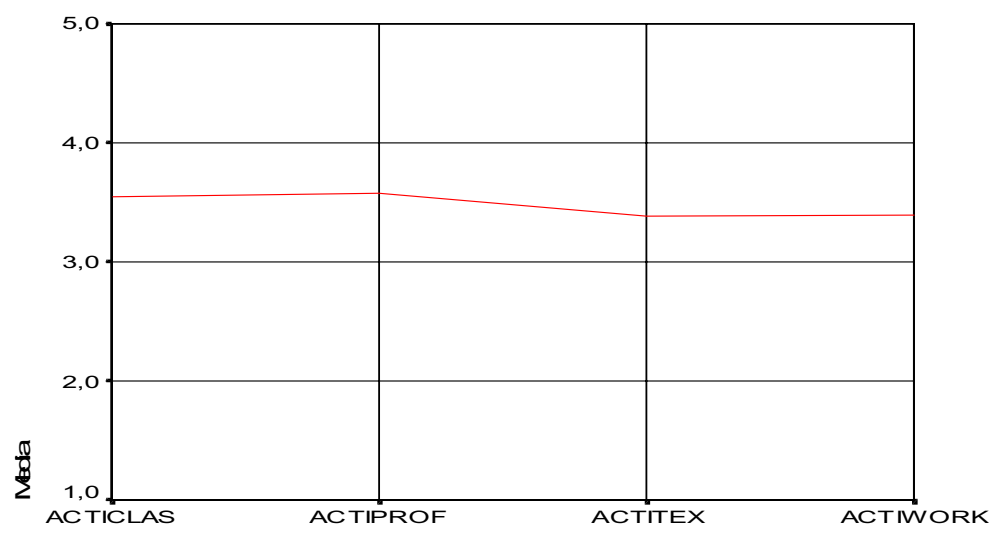

El contraste entre los valores medios de las cuatro subescalas no nos proporciona grandes diferencias entre ellas (ver Tabla 2), a partir del cálculo del estadístico descriptivo Tamaño del Efecto. Esto nos da a entender que la percepción de los diferentes factores es homogénea y que, en general, ninguno de ellos destaca de manera especial sobre los demás.

Tabla 2. Contraste entre los valores medios de las cuatro subescalas.

\begin{tabular}{llc}
\hline \multicolumn{1}{c}{ Variables a comparar } & $\mathrm{P}$ & Decisión \\
\hline & & \\
ACTICLAS versus ACTIPROF & .10 & No hay diferencias \\
ACTITEXT versus ACTIWORK & .12 & No hay diferencias \\
ACTICLAS versus ACTIWORK & .25 & No hay diferencias \\
ACTIPROF versus ACTITEXT & .28 & No hay diferencias \\
ACTIPROF versus ACTIWORK & .29 & No hay diferencias \\
ACTICLAS versus ACTITEXT & .34 & No hay diferencias
\end{tabular}

La actitud que muestra este grupo español hacia el profesorado comparte criterios con la actitud que muestran hacia el libro de texto, hacia la clase en general y hacia las actividades, tareas, y ejercicios que en ella se desarrollan. Esto es, en cierta manera, lógico, si pensamos en la simetría que guardan las cuatro subescalas respecto a los constructos que mide cada una de ellas (contenidos, juegos, materiales,...).

A continuación, se aplicó el Test de Levene a las cuatro subescalas como prueba previa para verificar si procedía o no el uso de los modelos de análisis de la varianza. Para ello, se comprobó la homogeneidad de las varianzas de las cuatro subescalas; como los valores obtenidos son todos superiores a 0,05 , podemos asumir que no existen diferencias significativas entre las varianzas y podemos, por tanto, aplicar este modelo de análisis. 
Tabla 3. Prueba de homogeneidad de varianzas.

\begin{tabular}{lcccc}
\hline & $\begin{array}{c}\text { Estadístico } \\
\text { de Levene }\end{array}$ & g11 & g12 & Sig. \\
\hline & & & & \\
ACTIPROF & 0.96 & 1 & 177 & .32 \\
ACTICLAS & 1.23 & 1 & 177 & .26 \\
ACTICTEXT & 0.15 & 1 & 177 & .69 \\
ACTIWORK & 0.03 & 1 & 177 & .85 \\
\hline
\end{tabular}

También se verificó el supuesto de normalidad de la distribución de los datos. Se comprobó si la distribución se ajustaba a la curva normal, aplicando como prueba de normalidad el Test de Kolmogorov-Smirnov. Obtuvimos los siguientes valores:

Tabla 4. Prueba de normalidad Kolmogorov-Smirnov.

\begin{tabular}{lccc}
\hline & $\begin{array}{c}\text { Estadístico } \\
\text { Kolmogorov-Smirnov }\end{array}$ & gl & Sig. \\
\hline & & & \\
ACTIPROF & 0.07 & 177 & .013 \\
ACTICLAS & 0.08 & 177 & .002 \\
ACTICTEXT & 0.08 & 177 & .004 \\
ACTIWORK & 0.07 & 177 & .030 \\
\hline
\end{tabular}

Las cuatro subescalas cumplieron satisfactoriamente el supuesto de normalidad y, por tanto, la aplicación de los análisis de la varianza (ANOVA) era pertinente.

\subsection{Análisis de la varianza}

Se llevaron a cabo repetidos análisis de la varianza con las diferentes subescalas a partir de las respuestas del alumnado hacia el inglés como idioma extranjero, tomando como variables independientes el sexo (chicas, $\mathrm{n}=89$; chicos, $\mathrm{n}=87$ ), la clase social (Baja, $\mathrm{n}=44$; Media-Baja, $\mathrm{n}=56$; Media, $\mathrm{n}=33$ ), el curso de iniciación al idioma (Tercero, $\mathrm{n}=8$; Cuarto, $\mathrm{n}=3$; Quinto, $\mathrm{n}=11$; Sexto, $\mathrm{n}=126$; Primero ESO, $\mathrm{n}=3$; Segundo ESO, $\mathrm{n}=1$ ), la nota media en la clase de inglés en años anteriores (Insuficiente, $n=6$; Suficiente, $n=54$; Bien, $\mathrm{n}=64$; Notable, $\mathrm{n}=29$; Sobresaliente, $\mathrm{n}=14$ ) y la asistencia a clases particulares (los que habían recibido clases particulares, $\mathrm{n}=50$ y los que no habían recibido clases particulares, $\mathrm{n}=83$ ).

La Tabla 5 presenta los resultados de los ANOVAS. El análisis de la varianza indica que:

- Variable Género/Sexo. Hay diferencias estadísticamente significativas entre las actitudes hacia las clases de inglés con respecto a la variable género; las chicas tienen una actitud más favorable que los chicos. No se encontraron, sin embargo, diferencias en las tres subescalas restantes. 
- Variable Clase Social. No se encontraron diferencias estadísticamente significativas.

- Variable Curso de Iniciación. Se encontraron diferencias significativas en las subescalas de actitud hacia el trabajo y las tareas de clase. Los que se iniciaron más pronto en el aprendizaje del inglés tienen actitudes más favorables que los que se iniciaron más tarde.

- Variable Nota Media. No se encontraron interacciones significativas en ninguna de las cuatro subescalas. La nota media no discrimina la actitud de este grupo español hacia el aprendizaje del inglés.

- Variable clase social. El análisis de los resultados no revela interacciones importantes.

Tabla 5. Resultados según las variables independientes

\begin{tabular}{lccccccccccc}
\hline & Género & \multicolumn{1}{c}{ Clase social } & \multicolumn{2}{c}{$\begin{array}{c}\text { Curso de } \\
\text { iniciación }\end{array}$} & Nota media & \multicolumn{2}{c}{$\begin{array}{c}\text { Clases } \\
\text { particulares }\end{array}$} \\
\hline & F & Sig. & F & Sig. & F & Sig. & F & Sig. & F & Sig. \\
\hline & & & & & & & & & & \\
ACTICLAS & 4.56 & $.03^{* *}$ & 1.44 & .23 & 2.09 & .06 & 2.20 & .07 & .13 & .71 \\
& & & & & & & & & & \\
ACTIPROF & 3.05 & .08 & 2.08 & .10 & 1.09 & .36 & 1.11 & .35 & .09 & .75 \\
& & & & & & & & & & \\
ACTITEXT & 1.32 & .24 & 1.14 & .33 & 1.86 & .10 & 0.65 & .62 & .40 & .52 \\
& & & & & & & & & & \\
ACTIWORK & 2.77 & .10 & 0.68 & .56 & 2.71 & $.02 * *$ & 1.39 & .24 & .05 & .81 \\
& & & & & & & & & & \\
\hline
\end{tabular}

\section{Discusión}

Aunque las limitaciones del estudio debido a la muestra tan pequeña de discentes analizados son importantes, hay algunos hallazgos que merece la pena destacar. Esta muestra española parece que no siente como necesidad vital el estudio de un idioma extranjero. Sus actitudes se sitúan en unos valores medios entre lo indiferente y lo positivo, con una inclinación ligera a lo segundo, ya que, posiblemente, el entorno exterior y sus motivaciones personales hacen que el inglés no sea un elemento prioritario en sus vidas (Gardner, 2007). Llegan al instituto y se encuentran con un currículo oficial en el que tienen que cursar una serie de materias; quizá el margen de elección entre francés o inglés condicione una cierta predisposición hacia una segunda lengua, que en el caso del inglés se ha venido convirtiendo en un idioma hegemónico. Esta hegemonía del inglés hace que se tenga hacia su estudio actitudes más cercanas a lo positivo que a lo indiferente, aunque algunas veces también se percibe como una amenaza (Hilgendorf, 2007, Lasagabaster, 2005b). Para ver la importancia del inglés en Europa, señalar el hecho de que más del 38 por ciento de los ciudadanos lo 
habla como idioma extranjero y en España un 85 por ciento de las familias españolas considera que sus hijos e hijas debieran aprenderlo (Comisión Europea, 2006). Sin embargo, su aprendizaje parece que no es imprescindible, sino algo complementario que puede servir de ayuda en un futuro y que, a corto plazo, no va a influir en su modo de vida. Esta escasa motivación se pone de manifiesto cuando el alumnado entra en la universidad. Según Ruíz Garrido (2007), el nivel de competencia oral de los recién llegados está muy por debajo de lo deseado.

Habrá que determinar en estudios siguientes la percepción inicial de que el alumnado español parece que ha desarrollado actitudes ligeramente positivas que propician o favorecen la aparición de una motivación instrumental a la hora de aprender un idioma extranjero. $\mathrm{Su}$ deseo de adquirir una segunda lengua se limita a la obtención de unos fines prácticos, bien sea utilizar el otro idioma como una herramienta de estudio, bien para conseguir un trabajo mejor. Esto hace que sus actitudes sean menos positivas que las de aquellos grupos que estudian una segunda lengua con el deseo de formar parte de la comunidad cuya lengua están aprendiendo (Gardner, 1985). En este sentido, Brutt-Griffler (2002) distingue entre «inglés como idioma auxiliar» e «inglés como idioma internacional». El primero sirve de medio de comunicación interno en una sociedad plurilingüe -como Nigeria-, y el segundo denota escaso deseo de formar parte de la otra cultura -como en España-. Es necesario, según el citado autor, apreciar la cultura de uno de los países donde se habla el inglés para aprender a utilizarlo efectivamente. De lo contrario, estamos avocados a lo que Hilgendorf (2007) denomina «EuroEnglish»; es decir, al inglés estándar que se habla en cada país.

La muestra española se ha llevado a cabo en comunidades monolingües (Andalucía y Murcia) donde las oportunidades de utilizar el otro idioma son escasas. Por tanto, el alumnado no encuentra un estímulo en su entorno que lo motive a utilizar el inglés. Como la práctica de esa segunda lengua se limita casi al ámbito escolar, posiblemente las actitudes de este grupo están directamente relacionadas con las experiencias que tienen en el aula.

Esto explicaría los resultados obtenidos a partir del análisis de la varianza sobre el poder moderador de algunas variables independientes en las actitudes del alumnado. No se han encontrado grandes diferencias en función de estas características. Tan sólo las chicas han mostrado actitudes más positivas que los chicos hacia las actividades de clase y que aquellos alumnos que comienzan a estudiar el inglés antes tienen actitudes más favorables hacia las tareas de clase. Quiere esto decir que, en general, el grupo analizado es muy compacto y que tiene una visión del aprendizaje de inglés muy parecida. Las diferencias de estos resultados con estudios anteriores (Gardner, 1985), que encontraron interacciones significativas entre varias de las variables independientes aquí estudiadas y los índices actitudinales, se explican, como decíamos anteriormente, aludiendo al contexto tan diferente donde esos estudios se llevaron a cabo y el contexto donde se ha desarrollado el nuestro. En España el contacto con hablantes nativos de habla inglesa es muy limitado -hay poco más de 160 mil residentes, según un boletín del Instituto Nacional de Estadística en 2004-, mientras que la mayoría de los estudios en el campo del aprendizaje de segundos idiomas (ver Masgoret y Gardner, 2003, para un detallado análisis) tuvieron lugar en Canadá y Estados Unidos donde la presencia de hablantes del idioma analizado era, obviamente, muy importante. 


\section{RECOMENDACIONES}

La amplia presencia del inglés en la mayoría de las universidades europeas nos invita a reflexionar sobre el desarrollo de políticas pedagógicas coherentes que recaben el conocimiento y la experiencia del profesorado de inglés y expertos en el área de planificación (Lasagabaster, 2002). Será conveniente también tener en cuenta una serie de condiciones que garanticen la calidad de la enseñanza de la lengua extranjera: el tiempo dedicado, los objetivos lingüísticos, la metodología y la formación del profesorado, entre otros (Cenoz, 2003). Asimismo, debería alentarse al alumnado a conocer de manera más profunda la comunidad y los hablantes del otro idioma, ya que el contacto frecuente parece que promueve actitudes favorables (Dörnyei, 2006; Dörnyei y Csizér, 2005; Gardner, 2007; Pettigrew y Tropp, 2004). Actividades como los viajes de estudios, intercambios o inmersiones vía música, televisión o cine pueden ayudar a comprender el contexto donde el aprendizaje del inglés toma cuerpo. Algunos autores como Dörnyei y Csizér (2005) recomiendan que se promuevan los contactos entre culturas a nivel nacional. Las excursiones, por ejemplo, se han hecho muy populares en los últimos tiempos en España. Se trata, entonces, de explicar convenientemente los objetivos y la influencia que pueden tener desde un punto de vista social y, sobre todo, educativo. De igual manera, recomendamos el uso de subtítulos para entender mejor las películas en inglés. Como queda demostrado, los países que utilizan este recurso a nivel nacional están entre los Estados Miembros donde los ciudadanos hablan más de un idioma (Comisión Europea, 2006).

Finalmente, aconsejamos que las distintas administraciones educativas incluyan el estudio de la lengua extranjera a la edad más temprana posible. La Commission of the European Languages en 2005 animó a promover el estudio de un idioma extranjero en todos los países miembros cuanto antes. Con este fin, se diseñó un plan estratégico que preparase adecuadamente a los maestros. Esta es una idea interesante que podría tener un efecto muy positivo en las actitudes del alumnado más reacio al inglés. Las posibilidades de ser completamente bilingües son mayores cuanto antes se empiece a estudiar el segundo idioma. Además, y como queda demostrado en numerosos estudios, el «bilingüismo facilita el aprendizaje de una lengua adicional», ya que la «exposición a dos lenguas contribuye a desarrollar una mayor conciencia metalingüística, es decir, mayor capacidad para analizar la lengua como objeto de reflexión en sí mismo» (Lasagabaster, 2005a: 418-419).

\section{REFERENCIAS BIBLIOGRÁFICAS}

Agulló, G. (2006). «Overcoming age-related differences», en ELT Journal, 60, 4: 365-373.

Antes, T.A. (1999). «But will I ever use this foreign language? Student perception of the applicability of foreign language skills», enForeign Language Annals, 32, 2: 219-233.

Arsenian, S. (1945). «Bilingualism in the post-war world», en Psychologica Bulletin, 42, 6586.

Artigal, J.M., Arizmendi, J. y Azpillaga, B. (eds.)(1996). Enseñar y aprender una lengua extrajera en el segundo ciclo de la educación infantil. Barcelona: Asociación de Maestros Rosa Sensat.

Bialystok, E. (1978). The teaching and learning of French as a second language in two distinct learning settings. Toronto: Ontario Institute for Studies in Education. 
Brutt-Griffler, J. (2002). World Englishes: a study of its developments. Clevelon: Multilingual Matters.

Cenoz, J. (2003). «El aprendizaje del inglés desde educación infantil: efectos cognitivos, lingüísticos y afectivos», en Eduling, 1. Descargado el 18 de noviembre del 2007 de http://www.ub.es/ ice/portaling/eduling/esp/n_1/cenoz-art.htm.

Comisión Europea (2006). Especial Eurobarómetro: Los Europeos y sus idiomas. Descargado el 17 de noviembre del 2007 de http://www.ec.europa.eu/public_opinion/archives/ebs/ ebs_243_en .pdf.

Commission of the European Languages (2005). A new framework strategy for multilingualism. Descargado el 18 de noviembre del 2007 de http://ec.europa.eu/education/policies/lang/ doc/com596_en.pdf.

Dörnyei, Z. (2006). «Individual differences in second language acquisition», en AILA Review, 19, 42-68.

Dörnyei, Z. y Csizér, K. (2005). «The effect of intercultural contact and tourism on language attitudes and language learning motivation», en Journal of Language and Psychology, 24, 4: 327-357.

Eccles, J. y Wigfield, A. (1985). «Teacher expectations and student motivation. En J. B. Dusek (eds.), Teacher Expectancies (pp. 185-226). Hillsade, NJ: Erlbaum.

Gálvez Díaz, D.J. (2001). Enseñanza del inglés en segundo ciclo de educación infantil. Mérida: Junta de Extremadura, Dirección General de Ordenación, Renovación y Centros.

Gardner, R.C. (1985). Social psychology and second language learning: the role of attitudes and motivation. London: Edward Arnold.

Gardner, R.C. (2007). «Motivation and second language acquisition», en Porta Linguarum, 8, 525.

Gardner, R.C. y MacIntyre, P. (1993). «On the measurement of affective variables in second language learning», en Language Learning, 43, 157-194.

Gardner, R.C., Masgoret, A.M., Tennant, J. y Mihic, L. (2004). «Integrative motivation: changes during a year-long intermediate- level language course», en Language Learning, 5, 1: 1-34.

Good, T.L. y Brophy, J.E. (eds.)(2003). Looking in classrooms (9th ed.). Boston: Allyn \& Bacon.

Hosenfeld, C. (1978). «Student's mini-theories of second language learning», en Association Bulletin, 28, 2-10.

Hilgendorf, S.K. (2007). «Symposium on the Englishes of Europe in the new millennium: introduction», en World Englishes, 26, 2: 107-110.

Instituto Nacional de Estadística (Marzo, 2004). Boletín Informativo. Descargado el 16 de noviembre del $2007 \mathrm{de}$ http://www.ine.es/revistas/ cifraine/cifine_ext0604.pdf.

Jakobobitz, L.A. (1970). Foreign language learning: a psycholinguistic analysis of the issues. Rowley: Newbury House Publishers.

Jussim, L., Smith, A., Madon, S. y Palumbo, P. (1998). «Teacher expectations», en J. E. Brophy (eds.), Advances in research on teaching: Expectations in the classroom (Vol. 7, pp.148). Greenwich, CT: JAI Press.

Lambert, W.E. (1955). «Measurement of the linguistic dominance of bilinguals», en Journal of Abnormal and Social Psychology, 50, 197-200.

Lasagabaster, D. (2002). "The role of instrumental and integrative attitudes in a multilingual context», en II Simposio Internacional del Bilingüismo. Victoria-Gasteiz: Universidad del País Vasco. 
Lasagabaster, D. (2005a). «La presencia de tres lenguas en el currículo:multilingualismo en los contextos canadiense y español», en Revista de Educación, 337, 405-426.

Lasagabaster, D. (2005b). "Attitudes towards Basque, Spanish and English: An analysis of the most influential variables», en Journal of Multilingual and Multicultural Education, 26, 4: $296-315$.

Littlewood, W. (2001). «Student's attitudes to classroom English learning: a cross-cultural study», en Language Teaching Research, 5, 1: 3-28.

Madrid, D. (1999). La investigación de los factores motivacionales en el aula de idioma. Granada: Grupo Editorial Universitario.

Mantle-Bromley, C. (1995). «Positive attitudes and realistic beliefs: links to proficiency», en The Modern Language Journal, 79, 3: 372-386.

Manzaneda, F. y Madrid, D. (1997). «Actitudes y motivación en la clase de inglés (1 de BUP), en E. A. Adams, A. Bueno y G. Tejada (eds.)(1997): Francisco Manzaneda Oneto: In Memoriam», The Grove, 4, 153-171.

Masgoret, A.M. y Gardner, R.C. (1999). «A causal model of Spanish immigrant adaptation in Canada», en Journal of Multilingual and Multicultural Development, 20, 3: 216-235.

Masgoret, A.M. y Gardner, R.C. (2003). «Attitudes, motivation, and second language learning: a meta-analysis of studies conducted by Gardner and associates», en Language Learning, 53, 1: 167-210.

Mur, O. (1998). «¿Cómo introducir el inglés en educación infantil?», en Escuela Española, 4, 23-30.

Pellegrini, A.D. y Blachtford, P. (eds.)(2000). The child at school: Interactions with peers and teachers. London: Arnold.

Ruiz Garrido, M. (2007). «Teaching and learning English for business communication: a case in Spain», en Business Communication Quarterly, 70, 1: 74-79.

Rodríguez López, B. y Varela Méndez, R. (2004). «Models of teaching foreign languages to young children», en Didáctica (Lengua y Literatura), 16, 163-175.

Rodríguez Sánchez, M.T. y McCarthur, F. (eds.) (2001). Lenguas extranjeras en la etapa de educación infantil. Mérida: Junta de Extremadura, Dirección General de Ordenación, Renovación y Centros.

Rubie-Davies, C.M. (2006). «Teacher expectations and students self-perceptions: exploring relationships», en Psychology in the Schools, 43, 5: 537-552. 


\section{APÉNDICE A. 1 \\ Cuestionario sobre actitudes}

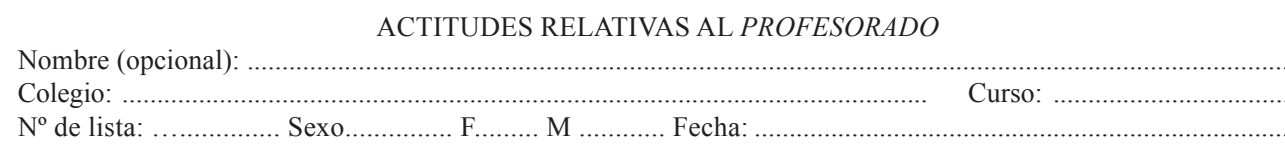

¿Cuál es tu actitud respecto a la actuación didáctica de tu profesorado en el aula; respecto a su forma de dar las clases, tratar a los y las estudiantes, etc...? Lee y escribe el número que mejor indique tu actitud y explica brevemente por qué motivo reaccionas así. Ten en cuenta que:

\section{0=Muy negativa $\quad 1=$ Negativa $\quad 2=$ Indiferente $\quad 3=$ Positiva $\quad 4=$ Muy positiva}

1. Mi actitud e interés hacia su forma de dar los contenidos. ( ) Comentario

2. Mi actitud e interés hacia su forma de comportarse ante las necesidades e intereses de los estudiantes. () Comentario

3. Mi actitud e interés hacia su forma de explicar. ( ) Comentario

4. Mi actitud e interés hacia su forma de preparar las clases. ( ) Comentario

5. Mi actitud e interés hacia su forma de favorecer la participación de los y las estudiantes. ( ) Comentario

6. Mi actitud e interés hacia su motivación y entusiasmo para la enseñanza ( ) Comentario

7. Mi actitud e interés hacia los materiales del profesorado. ( ) Comentario

8. Mi actitud e interés hacia su forma de motivar a los y las estudiantes. ( ) Comentario

9. Mi actitud e interés hacia su forma de dar las clases: presentación y trabajo con los materiales, forma de evaluar, etc... ( )

Comentario

10. Mi actitud e interés hacia su forma de evaluar. ( ) Comentario

11. Mi actitud e interés hacia el balance entre el trabajo oral y el escrito. ( ) Comentario

12. Mi actitud e interés hacia su forma de explotar los materiales audiovisuales. ( ) Comentario.

12. Mi actitud e interés hacia su forma de tratar al alumnado ( ) Comentario

13. Mi actitud e interés hacia su forma de enfocar los aspectos sociolingüísticos y socioculturales. ( ) Comentario

14. Mi actitud e interés hacia su forma de mantener la disciplina en clase. ( ) Comentario

15. Mi actitud e interés hacia su forma de organizar el trabajo individual, en parejas y en grupo. ( ) Comentario

16. Mi actitud e interés hacia su forma de organizar juegos/actividades similares. ( ) Comentario 


\section{ACTITUDES RELATIVAS AL LIBRO DE TEXTO}

Nombre (opcional):

Colegio:

$\mathrm{N}^{\mathrm{o}}$ de lista:

Sexo........

F......... M

Curso:

¿Cuál es tu actitud respecto al libro de texto: graduación, ilustraciones, enfoque de las unidades didácticas, etc...?

Lee y escribe el número que mejor indique tu actitud. Ten en cuenta que:

\section{0=Muy negativa $\quad 1=$ Negativa $\quad 2=$ Indiferente $\quad 3$ =Positiva $\quad 4=$ Muy positiva}

1. Mi actitud e interés hacia la encuadernación, el tamaño del libro, espacios, papel, dibujos, letra e ilustraciones.

2. Mi actitud e interés hacia la forma de presentar los contenidos que estudiamos.

3. Mi actitud e interés hacia la estructuración, presentación y desarrollo de las unidades didácticas del libro.

4. Mi actitud e interés hacia la conexión que establece el libro de texto con otras áreas y que permite aprender sobre otros países, educación vial y sobre la naturaleza.

5. Mi actitud e interés hacia la variedad de ejercicios que tiene el libro.

6. Mi actitud e interés hacia los juegos y canciones del libro.

7. Mi actitud e interés hacia los repasos del libro.

8. Mi actitud e interés hacia los esquemas, tablas, resúmenes y cuadros sinópticos del libro.

9. Mi actitud e interés hacia los diálogos del libro.

10. Mi actitud e interés hacia las actividades de conversación del libro.

11. Mi actitud e interés hacia las actividades escritas del libro de ejercicios.

12. Mi actitud e interés hacia los textos de lectura que tiene el libro.

13. Mi actitud e interés hacia los 'listenings' del libro.

14. Mi actitud e interés hacia los ejercicios de vocabulario del libro.

15. Mi actitud e interés hacia las actividades de fonética del libro.

16. Mi actitud e interés hacia las actividades de cultura y civilización del libro.

\section{ACTITUDES RELATIVAS A LAS TAREAS EJERCICIOS DE CLASE}

Nombre (opcional):

Colegio:

$\mathrm{N}^{\mathrm{o}}$ de lista:

Sexo..

F......... M Fecha:

Curso:

¿Cuál es tu actitud respecto a las tareas y ejercicios que hacéis en la clase de inglés? Lee y escribe el número que mejor indique tu actitud. Ten en cuenta que:

\section{0=Muy negativa $\quad 1=$ Negativa $2=$ Indiferente $\quad 3=$ Positiva $\quad 4=$ Muy positiva}

1. Mi actitud e interés hacia los juegos y canciones.

2. Mi actitud e interés hacia las grabaciones en casete ('listening').

3. Mi actitud e interés hacia las grabaciones en vídeo adaptadas al nivel del alumnado.

4. Mi actitud e interés hacia los ejercicios orales entre estudiantes y profesorado.

5. Mi actitud e interés hacia las actividades de vocabulario (palabras estudiadas y nuevas).

6. Mi actitud e interés hacia las actividades por equipos.

7. Mi actitud e interés hacia las actividades individuales.

8. Mi actitud e interés hacia las actividades por parejas.

9. Mi actitud e interés hacia la dramatización y representación de diálogos y situaciones.

10. Mi actitud e interés hacia la forma de presentar los contenidos.

11. Mi actitud e interés hacia los ejercicios de pronunciación. 
12. Mi actitud e interés hacia los ejercicios de comprensión oral.

13. Mi actitud e interés hacia las actividades que requieren salir a la calle y recoger datos.

14. Mi actitud e interés hacia la estructuración, presentación y de las unidades didácticas del libro.

15. Mi actitud e interés hacia los ejercicios de gramática.

16. Mi actitud e interés hacia las actividades escritas.

17. Mi actitud e interés hacia las actividades de conversación.

18. Mi actitud e interés hacia las actividades de cultura y civilización. ( )

19. Mi actitud e interés hacia consultar folletos, sacar información, recortar

20. Mi actitud e interés hacia las actividades de lectura.

21. Mi actitud e interés hacia las actividades escritas del libro de ejercicios.

\section{ACTITUDES RELATIVAS A LA CLASE DE INGLES}

Nombre (opcional):

Colegio:

$\mathrm{N}^{\mathrm{o}}$ de lista: Sexo.

F......... Fecha:

Curso:

¿Qué actitudes tienes respecto a lo que haces en la clase de inglés? ¿Por qué motivo te has formado esa actitud? Lee y escribe el número que mejor indique tu actitud. Ten en cuenta que:

\section{0=Muy negativa $\quad 1=$ Negativa $\quad 2=$ Indiferente $\quad 3=$ Positiva $\quad 4=$ Muy positiva}

1. Mi actitud e interés hacia los contenidos en general que estudiamos en la clase de inglés.

2. Mi actitud e interés hacia los materiales que usamos en la clase de inglés (libros, cintas, vídeos, libro de ejercicios, etc...).

3. Mi actitud e interés hacia las explicaciones del profesorado.

4. Mi actitud e interés hacia la metodología del profesorado.

5. Mi actitud e interés a la hora de participar en clase.

6. Mi actitud e interés hacia las actividades escritas.

7. Mi actitud e interés hacia las actividades orales.

8. Mi actitud e interés hacia las actividades individuales.

9. Mi actitud e interés hacia el trabajo en equipo.

10. Mi actitud e interés hacia el trabajo en parejas.

11. Mi actitud e interés hacia las correcciones.

12. Mi actitud e interés hacia las medidas de disciplina que se adoptan en clase.

13. Mi actitud e interés hacia la forma de evaluar del profesorado.

14. Mi actitud e interés hacia las canciones y los juegos.

15. Mi actitud e interés hacia los deberes.

16. Mi actitud e interés hacia el aula donde se imparten las clases.

17. Mi actitud e interés hacia la forma de pronunciar del profesorado.

18. Mi actitud e interés hacia el ritmo de la clase.

19. Mi actitud e interés hacia los compañeros.

\section{DATOS PERSONALES}

Nombre (opcional):

Colegio:

$\mathrm{N}^{\mathrm{o}}$ de lista: Sexo........ F... $\mathrm{M}$ Fecha:

Curso:

Por favor responde a lo siguiente:

Haz un círculo o una cruz donde consideres oportuno. 
1. El inglés:
A) Me gusta
B) Me gusta sólo un poco
C) No me gusta nada

2. Profesión del padre

3. Profesión de la madre

4. ¿En qué curso o grado comenzaste el estudio del inglés?

5. ¿Has recibido alguna vez clases particulares de apoyo o refuerzo?

6. Mi nota media en inglés viene siendo 